Proceedings of the Online Conference "Applications of Physics in Mechanical and Material Engineering"

\title{
Physical Acoustics Analysis of Composite Wooden Panel and Room by Computational Modeling
}

\author{
Ö. KARAÇALI* \\ Istanbul University-Cerrahpaşa, 34320 Avcılar, Istanbul, Turkey \\ Doi: 10.12693/APhysPolA.139.483 \\ *e-mail: ozdogank@istanbul.edu.tr
}

\begin{abstract}
This research is to analyze the factors that cause the distortion of the sound and to make the sound more propagable with the use of wooden acoustic panels (WAP) by the finite element analysis (FEA) in a closed room. The structural modeling-FEA has become the leading technique with the fluid-structure interaction (FSI) used to systematically analyze multi-physics events of how fluids and structures interact for acoustics as well as to solve structural problems for reverberation — sound pressure problems. The displacement-based nonlinear Lagrangian method was used for the acoustic fluid and material structure interaction behavior by FEA to manage the problem of FSI. Under laboratory conditions, the acoustic response of a composite WAP (ASTM E-84) and ceiling was experimentally studied and compared by computational models. This research enabled the designer to create a three-dimensional acoustic model of a WAP with full acoustic qualities for all surfaces to enhance the sound transmission by ANSYS-v.2020. The promising results showed that the consideration of the dominant fluid-fiber direction and sound absorption is of importance.
\end{abstract}

topics: acoustic analysis, wooden panels, sound absorption, finite element analysis

\section{Introduction}

Architectural acoustics are particularly concerned with the generation and propagation of sound [1] that starts to resonate, becoming clearly inaudible and uncomfortable [2]. At this point, the selection of finishing materials becomes important depending on the space geometry and sound absorption capacity $[1,2]$. Sound pollution problems $[3,4]$, which can be overcome by WAP, are often caused by sound waves reflected [4] by walls or ceilings [5]. To determine the acoustic performance of a space, it is necessary to make a series of mathematical calculations $[5,6]$ using the acoustic analysis FEM-FSI methods $[7-9]$ to obtain more precise results by using geometric data, and acoustic pressure, velocity data [10]. The structural models offer the option to perform a range of acoustic analyses for reverberation, sound pressure and velocity tracing techniques [11].

This research is part of a study conducted by testing the acoustic sound insulation of a room covered with acoustic composite WAP (35 $\mathrm{mm}$ in height) (ASTM E-84) with a natural wood veneer finish in double V-groove for the ceiling and sidewalls subjected to the loud sound source. The Kundt impedance tube with $100 \mathrm{~mm}$ for the low frequency range, and $30 \mathrm{~mm}$ diameter for higher frequencies was used to measure the sound pressure, absorption coefficient in frequency range $50-6400 \mathrm{~Hz}$ in experimental laboratory conditions. The ASTM E2611 measurement method ASTM E1050 and ISO 10534 - two measurement methods were applied. A low frequency generator was plugged into the speaker of the Kundt tube. The exploring microphone was then connected to the data acquisition system and the oscilloscope. A composite WAP and acoustic core rubber (25 $\mathrm{mm}$ in height) of absorbing materials was used to analyze the acoustic performance of the model by the finite element method.

\section{Mathematical model}

The fluid-structure interaction (FSI) problem addresses fluid behaviors in this research. The fluid displacement is small while the interaction is significant. Here, the problem of structural behaviors influences pressures build-up and reactions in a reverberation room. Differential equation is as follows:

$$
\frac{\partial^{2} p}{\partial x^{2}}+\frac{\partial^{2} p}{\partial y^{2}}+\frac{\partial^{2} p}{\partial z^{2}}+\frac{1}{\bar{c}^{2}} \frac{\partial^{2} p}{\partial t^{2}}=0
$$

matching the pressure distribution $p$ in a compressible fluid subjected to a small amplitude motion. Coefficient $\bar{c}$ represents the sound speed in the fluid. 
The case of WAP-solid objects that face the forced motion is described with

$$
\frac{\partial p}{\partial n}=-\rho \frac{\partial^{2} U_{n}}{\partial t^{2}}
$$

where $U_{n}$ - the component displacement.

Now, the quasi-harmonic equation in the finite element may be converted to the matrix differential equation, i.e.,

$$
G \ddot{p}+H p+\bar{f}_{f}=0,
$$

where $H$ and $G$ matrices are obtained from surface integrals associated with motions on the boundaries. This structural motion can be, in fact, discretized by a shape function

$$
U_{n}=\hat{N} \boldsymbol{a} \text {. }
$$

Its form is represented by $\hat{N}$ and the nodal parameters $\boldsymbol{a}$, known as displacement parameters. The physical forcing event $\bar{f}_{f}=S \ddot{\boldsymbol{a}}$ is given as an equation, while $S$ reads as

$$
S=\int_{\mathcal{S}} \mathrm{d} S N^{\mathrm{T}} \rho \hat{N},
$$

where $N$ defines the pressure distribution and $\mathcal{S}$ denotes the boundary conditions between the structure of the model and the fluid.

Now, the fluid pressure on the contact surface given as $\bar{f}_{s}$, the external forcing term represented by $\boldsymbol{r}$, the mass matrix $M$, the damping matrix $C$, and the stiffness matrix $K$ participate in the following relation:

$$
M \ddot{\boldsymbol{a}}+C \dot{\boldsymbol{a}}+K \boldsymbol{a}+\bar{f}_{s}+\boldsymbol{r}=0 .
$$

According to the above equations, the principle work may be applied to last forces, namely,

$$
\bar{f}_{s}=\int_{\mathcal{S}} \mathrm{d} S \hat{N}^{\mathrm{T}} p=\frac{1}{\rho} S^{\mathrm{T}} \boldsymbol{p} .
$$

Since pressure is given as $p=N \boldsymbol{p}$, finally we will obtain the coupled differential equations

$$
\begin{aligned}
& G \ddot{p}+H p+S \ddot{\boldsymbol{a}}=0 \\
& M \ddot{\boldsymbol{a}}+C \dot{\boldsymbol{a}}+K \boldsymbol{a}+\frac{1}{\rho} S^{\mathrm{T}} \boldsymbol{p}+\boldsymbol{r}=0
\end{aligned}
$$

which control the problem of FSI.

\section{Wooden acoustic panel by FEA and FSI}

The effects of wooden panels installation on sound behaviors are investigated with FEM to produce some guidelines on the design of acoustic frequency. The motion transmissibility at the center point of the panels predicted by FEM/FSI for the case study is shown in Fig. 1.

In this research, different acoustic pressure distributions and operating frequencies $(124-4000 \mathrm{~Hz})$ for acoustic core rubber inside a wooden panel are presented for absorption analysis in FSI as shown in Fig. 2. Here, in the finite element-FSI method, different colors show different sound frequency and pressure information. Therefore, the warmest color sounds represent the highest levels, while cool colors represent the lowest pressure $(1.5 \mathrm{~Pa}$ at $70.1 \mathrm{~dB})$.

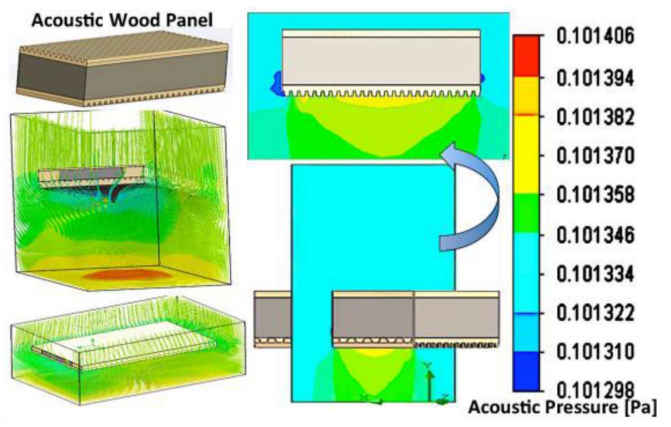

Fig. 1. Sound pressure in acoustic wood panel by FEA/FSI.
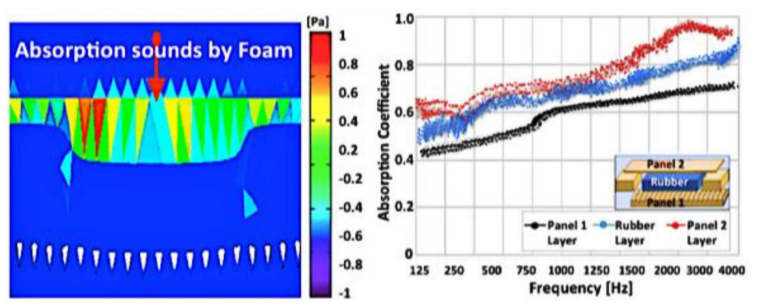

Fig. 2. Rubber material acoustic absorption analysis in FSI.

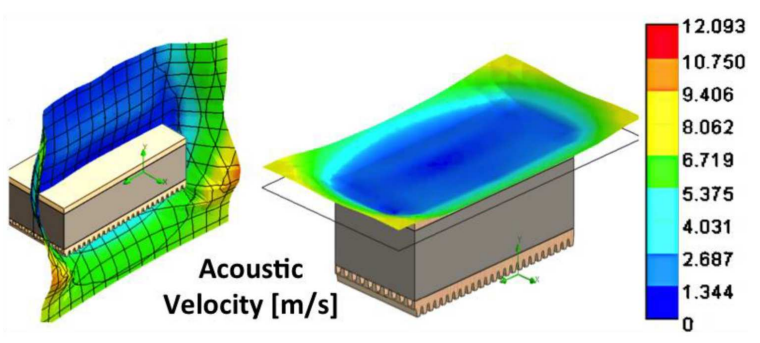

Fig. 3. Acoustic response in wood panel sample.

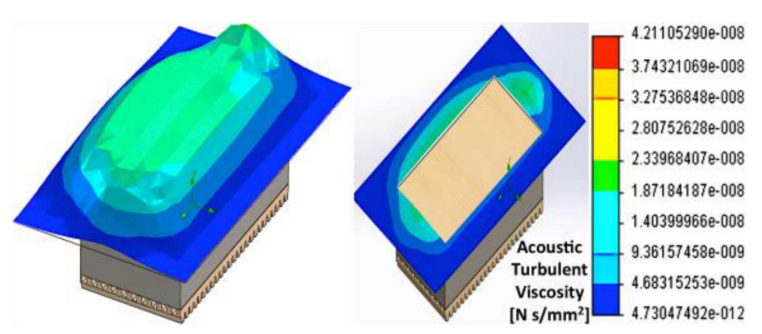

Fig. 4. Acoustic turbulent viscosity analysis of wood panel.

Acoustic response air-sound flow behavior in wood panel is presented for speed sound measured at $12.093 \mathrm{~m} / \mathrm{s}$ in Fig. 3. Acoustic wood panel absorption coefficient was computed in three-layer panel-WAP as shown in Fig. 4.

Due to a large number of cases, only groups corresponding to the 100 and $4000 \mathrm{~Hz}$ frequencies were taken into consideration in the acoustic turbulent viscosity analysis in Fig. 4. 


\section{Research}

Experimental tests were performed to verify the theoretical results in this research. The equipment setup with a wooden ceiling and a sidewall panel is illustrated in Fig. 5a while the boundary conditions are defined in Fig. 5b. Two high frequency loud speakers were located at the positions shown in Fig. 5c. The sound transmission measurements and analysis were performed on a WAP frame partition according to the ISO 10140 standard. The sound pressure level of a diffuse sound area in the room and in the receiving room was measured in one-third octave bands from $50 \mathrm{~Hz}$ up to $4000 \mathrm{~Hz}$.

The results of the simulation are shown in Fig. 6 . These are the detailed visuals of the sound levels and pressure values formed in the cross sections taken from the middle plane of the speaker (Fig. 6a), the face of the ceiling facing the room (Fig. 6b) by using the middle and the maximum noise lines of the ceiling insulation (Fig. 6c). A spatial average of the sound pressure levels was determined in the source and the receiving room by calculating the average across two microphone boom positions in the room, with an averaging period

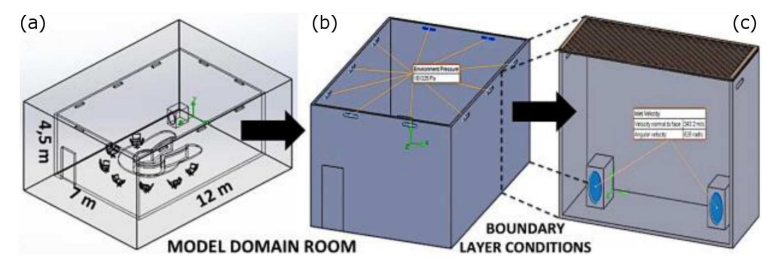

Fig. 5. Acoustic Computational Domain Meeting Room.

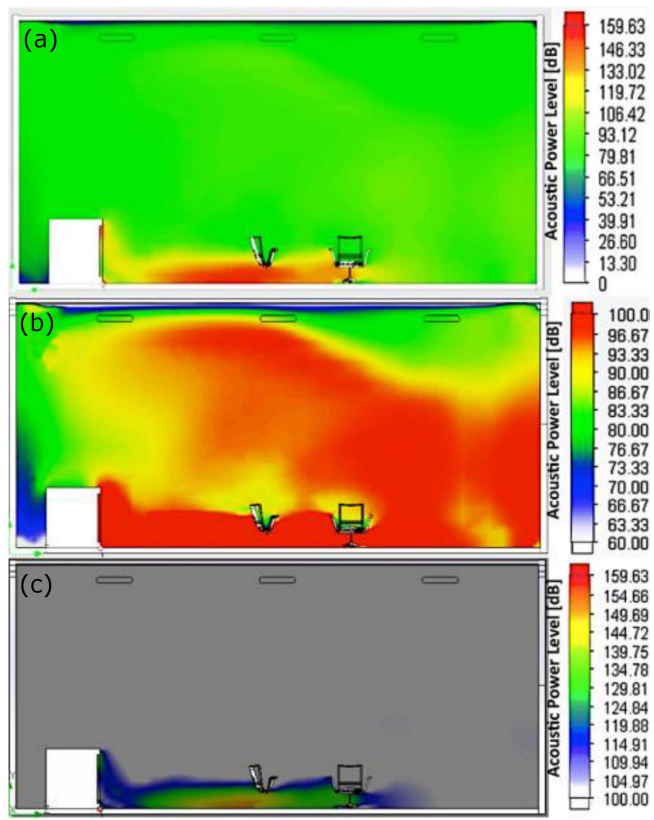

Fig. 6. Speaker Vertical Cross Section.
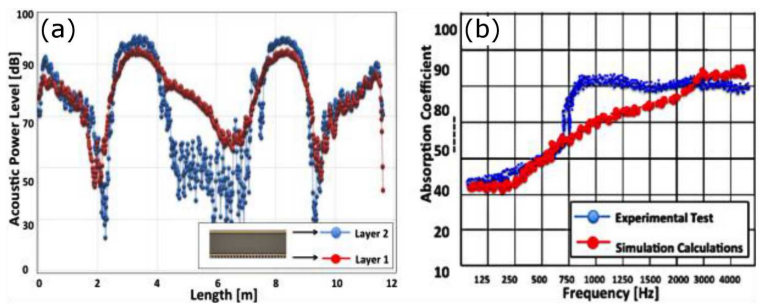

Fig. 7. Acoustic power absorption in wood panel room.

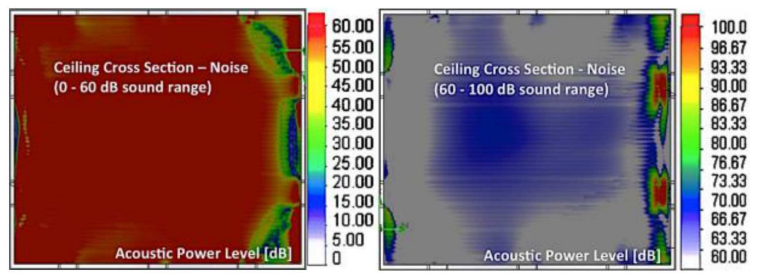

Fig. 8. Ceiling cross section acoustic model.

TABLE I

Composite wood panel noise reduction coefficient, where NCR - Noise Reduction Coefficient, SAA Sound Absorption Average. Composite dimensions: panel $-25 \mathrm{~mm}$, core $-35 \mathrm{~mm}$.

\begin{tabular}{c|c}
\hline \hline $\begin{array}{c}\text { Composite wood } \\
\text { panel thickness }\end{array}$ & Frequency $[\mathrm{Hz}]$ \\
\hline 0.07 & 125 \\
0.28 & 250 \\
0.85 & 500 \\
1.09 & 1000 \\
0.95 & 2000 \\
0.74 & 40000 \\
0.80 & NCR \\
0.79 & SAA
\end{tabular}

of $32 \mathrm{~s}$ at each boom position completed in one turn. The reverberation time of the receiving room in calculation of the sound reduction index- $R$ was measured based on the one-third octave band sound insulation values, and the weighted sound reduction index-RW, as a single number quantity, was determined according to ISO 717-1.

For a comparison of experimental tests and simulation study, the results of FEM-FSI and acoustic insulation index calculated by experimental tests are shown in Fig. 7. The data collected from the ceiling with the help of lines drawn in the maximum noise area of the noise in the ceiling regions of the two models are demonstrated in Fig. 7a. Here, both curves are a natural result of the symmetrical positioning of the two speakers in the room. It was observed that the three-layer model has created approximately $6 \mathrm{~dB}$ less noise at the maximum points than the single-layer model. Due to the average sound pressure level values in the center of 
the reverberation chamber, the sound insulation 42 and experimental results 41 were achieved. These respective - numerical and experimental - curves are encouraging results as presented in Fig. $7 \mathrm{~b}$.

The ceiling cross section acoustic simulation model at max. 60 and $100 \mathrm{~dB}$ range is shown in Fig. 8. Calculation sets were the following: the maximum loudness was measured as $149.3 \mathrm{~dB}$ in the loudspeaker section of the single layer model and $108.92 \mathrm{~dB}$ in the ceiling area of the threelayer model. The determined values are as follows: $159.63 \mathrm{~dB}$ in the speaker section and $103.02 \mathrm{~dB}$ in the ceiling section. The comparative results obtained using the middle and maximum noise lines of the ceiling insulation are given in Fig. 8. The measured composite wood panel noise reduction coefficient and sound absorption average are illustrated in Table I.

\section{Conclusions}

In this work, a computational simulation method was presented using the finite element model with FSI and applying the model itself to a composite panel made up of wood and rubber, with an internal frame, and experimental acoustic room model analysis. The tested models were subjected to real acoustic pressure as well as experimental study with Kundt tube tests required for determination of the models' natural periods and modes of vibration. The analytical part of the investigations included the definition of the physical model of the systems, and the determination of the constitutive nonlinear relationships for the contact zone between the panels and the sound foundations. Some peaks in graphs (Fig. 7a and b) are observed due to the resonance phenomenon. A reasonable correlation between the experimentally and numerically obtained displacements and acceleration at the top of the model has been obtained as shown in Fig. $7 \mathrm{~b}$.

\section{References}

[1] L. Savioja, U.P. Svensson, J. Acoust. Soc. Am. 138, 708 (2015).

[2] L. Alimonti, N. Atalla, J. Sound Vib. 367, 84 (2016).

[3] R.S. Puri, Appl. Math. Modell. 33, 4097 (2009).

[4] S. Garus, Rev. de Chim. 70, 3671 (2019).

[5] S. Garus, Acta Phys. Pol. A 138, 299 (2020).

[6] J. Zang, Front. Mech. Eng. 2, 62 (2007).

[7] A. Bella, M. Mitrovic, Sustainability 12, $5612(2020)$.

[8] X. Li, K. Yu, Appl. Acoust. 132, 82 (2018).

[9] K. Hun, IEEE/ACM Trans. Audio Speech Language Process. 23, 6 (2015).

[10] D.S. Williamson, IEEE/ACM Trans. Audio Speech Language Process. 25, 7 (2017).

[11] J. Piechowicz, I. Czajka, Archiv. Acoust. 37, 97 (2012). 